\title{
Evaluating Potential Customers' Perspectives for Success of Electric Vehicles in Poland: Fuzzy Multi-Criteria Decision Making Model
}

\author{
Shohreh Moradi ${ }^{1}$, Grzegorz Sierpiński ${ }^{2, *}$ \\ ${ }^{1}$ Iran University of Science \& Technology, Master of Science, Department of Railway Transportation Engineering \\ Hengam St., Resalat Sq., Tehran, IRAN \\ shohreh.moradi1372@gmail.com \\ ${ }^{2}$ Silesian University of Technology, Faculty of Transport and Aviation Engineering, Department of Transport Systems, \\ Traffic Engineering and Logistics \\ Krasińskiego Str. 8, 40-019 Katowice, POLAND \\ * Corresponding author: grzegorz.sierpinski@ polsl.pl
}

\section{Extended Abstract}

Electric vehicles (EVs) are widely regarded as among the most key technologies for addressing the global energy supply issue and pollution caused by fossil fuels. The aim of decreasing transportation-related emissions and phase-out of fossil-fuelled autos in (urban) transportation by 2050 offer unrivalled possibilities to promote electro-mobility. Electromobility is now a hot topic among EU institutions, national governments, city authorities, and even private customers. Despite the fact that the vision for electromobility's growth was outlined five years ago, electromobility in Poland is still in its infancy. Although the number of electric passenger cars on Polish roads is growing month by month, the prospects for achieving the government's target of one million electric vehicles by 2025 are slim. The main barriers are primarily the high price of an electric car and the still insufficient dedicated infrastructure. The greater number of charging stations is mainly found in larger cities, although it is also not sufficient. There is also a lack of fast charging stations on highways. This severely limits the planning of longer routes with the use of EV. Planning of a trip using EV is difficult as planning systems are usually limited to the grid of one selected energy supplier. Due to these and other problems, the increase in the number of EVs is still too slow.

This article provides a basis for computing a social, economic, and environmental index, which are based on customers' motivations to purchase electric vehicles, to address this issue. Selected research group of industry experts from Poland were chosen to validate the suggested research approach. Based on research, appropriate electric vehicles' social, economic, and environmental enablers, dimensions, and features were selected and verified through numerous rounds of discussion with industry professionals. The fuzzy TOPSIS method is more suitable to rank features according to customers' priorities. TOPSIS (Technique for Order of Preference by Similarity to Ideal Solution) is a multi-criteria decision-making technique. TOPSIS is built on the idea that the optimal choice is the one that has the smallest geometric distance from the positive ideal solution (PIS) and the largest geometric distance from the negative ideal solution (NIS). It's a compensating aggregation method for comparing a set of choices by giving each criterion a weight. The parameters or criteria in multi-criteria conditions are commonly in different dimensions, which can make evaluation challenging. As a result, to avoid this problem, a Fuzzy system is necessary. In TOPSIS, the criteria analysis process is simplified by the use of fuzzy numbers. As a result, Fuzzy TOPSIS is a simple, compensating approach and realistic model.

The study's findings will assist transportation industry managers, decision-makers, and government leaders in taking necessary steps to identify people's desires to buy electric vehicles. 